\title{
Validity of criteria for dementia in older people with intellectual disability
}

Andre Strydom* MBChB, MSc, MRCPsych, PhD

Trevor Chan MBChB MRCPsych MSc

Cormac Fenton MBChB MSc MRCPsych

Rebekah Craig DClinPsych

Gill Livingston MBChB, FRCPsych, MD

Angela Hassiotis MBChB, MA, MRCPsych, PhD

*corresponding author a.strydom@ucl.ac.uk ; UCL Department of Mental Health

Sciences, University College London, 67-78 Riding House Street, London W1W 7EY and Camden and Islington Foundation NHS Trust, London.

\section{Disclosures of interests}

All of the authors are employed either by University College London, or the NHS.

None of the authors have other interests to declare.

\section{Acknowledgments:}

The initial stages of the study was funded by the MRC through a Research Fellowship to Andre Strydom (G106/1160). The research was supported by the R\&D departments of Oxleas NHS Foundation Trust, Harrow Primary Care Trust (PCT), Enfield PCT, Islington PCT and Camden PCT, who all received a proportion of funding from the NHS Executive; the views expressed in this publication are those of 
Dementia criteria in intellectual disability

the authors and not necessarily those of the funders or the NHS Executive. We thank all the participants and their carers, and local area clinicians and social services staff involved in this study.

Keywords:

Dementia

Intellectual disability

Mental retardation

Diagnosis

Diagnostic criteria

Validity

Word count: 
Dementia criteria in intellectual disability

Validity of criteria for dementia in older people with intellectual disability

\section{Abstract}

Objective - Valid definitions of dementia should discriminate dementia from other forms of cognitive impairment such as Intellectual Disability (ID). We aimed to evaluate the usefulness of criteria for dementia and mild cognitive impairment (MCl) in ID, including predictive validity, and interrater reliability.

Method - We assessed 222 participants in a survey of older adults with ID without Down syndrome at two time points for dementia (T1 and T2). Mean follow-up period was 2.9 years. Dementia diagnoses were made according to ICD-10, DSM-IV, DCLD criteria. At follow-up (T2) raters were blind to initial diagnosis. Predictive validity was determined by comparing odds ratios of death, or of having a "poor outcome" (i.e. either dying or being diagnosed with dementia at T2).

Results - All dementia criteria showed substantial inter-rater reliability $(\kappa>0.68)$ and high specificity ( 95\%). Dementia cases at T1 were more likely to have died at T2 than those with no dementia (33.3\% vs $14.9 \%$; OR 2.85 ; $95 \% \mathrm{Cl} 1.12-7.22)$ and to have a "poor outcome" (77.8\% vs 27.6\%; OR 9.18; 95\% Cl $3.43-24.53)$. At least 2 dementia cases at $\mathrm{T} 1$ were false positives. Those with "MCl" at $\mathrm{T} 1$ were similar to "no dementia" cases in terms of poor outcomes at T2. .

Conclusions - Dementia diagnostic criteria show substantial reliability and satisfactory validity in ID. The diagnoses were, however, less stable than in the general population and some caution is advisable in those with more severe ID or additional sensory disability. $\mathrm{MCl}$ definitions require further consideration in the ID population.

Word count: 250 
Dementia criteria in intellectual disability

\section{Objective}

Valid definitions of dementia require the ability to discriminate it from other forms of cognitive impairment such as the pre-existing deficits in Intellectual Disability (ID). It is therefore crucial to examine the performance of dementia criteria in such populations, especially since lengthening life-expectancy has increased the likelihood of adults with ID developing dementia. Studies in the general population have shown that diagnostic criteria for dementia differ from each other, leading to different individuals being diagnosed (1-3). In adults with ID the problems in employing existing criteria are compounded by pre-morbid cognitive deficits and the variable quality of collateral information, and the validity and reliability of dementia criteria have not been examined in these older adults.

There has also been an increasing interest in mild cognitive impairment $(\mathrm{MCl})$ and its relationship to dementia. $\mathrm{MCl}$ has been proposed as a transitional stage between normal functioning and dementia $(4,5)$. However, it is not easy to employ definitions of $\mathrm{MCl}$ in people with ID due to pre-existing impairments.

In this study we aimed to evaluate the predictive validity and reliability of the ICD-10 (6) DSM-IV (7) and DC-LD (8) dementia criteria when applied in a one-off assessment (i.e. cross-sectionally) to older adults with intellectual disability. We also investigated whether a definition of ' $\mathrm{MCl}$ ' for this population is analogous in prognosis to definitions of $\mathrm{MCl}$ used in the general population.

Our main objective was to evaluate predictive validity of cross-sectional dementia diagnoses in the absence of post-mortem neuropathological data. In previous studies it has been shown that dementia is an independent predictor of death (9), with mortality rates far exceeding that of non-demented matched peers (10). Those that 
Dementia criteria in intellectual disability

have not died are expected to continue to be recognised as dementia cases and to deteriorate over time. In addition to dementia status, death or clinical deterioration is therefore used to evaluate diagnostic instruments $(11,12)$.

\section{Methods}

The appropriate Research Ethics Committee and Research and Development offices approved this study which followed up the participants in the Becoming Older with Learning Disability (BOLD) Memory Study (13, 14); a two-staged epidemiological survey of adults with non-Down syndrome intellectual disability aged $\geq 60$ years living in five London boroughs. As in the baseline interview (T1), the follow up (T2) consisted of a screen for dementia or cognitive decline. Those who screened positive had a full diagnostic assessment for dementia.

\section{Participants}

Potential participants in the initial BOLD Study were identified from ID teams and residential and day services providers. Participants included those resident in their own, family, residential or nursing homes and hospitals. Intellectual disability was defined according to ICD-10 criteria for mental retardation (6). Adults with Down syndrome were excluded from the study due to their known risk for Alzheimer's disease, and atypical presentation. 222 participants participated in the original study between 2004 and 2005 (T1). Of those, 60 had screened positive for dementia, of whom 28 had met at least one of $I C D-10, D S M-I V$ or $D C-L D$ sets of dementia criteria.

Potential participants at follow up (T2) were the original 222 participants. Written informed consent was obtained from those who had capacity to decide on their 
Dementia criteria in intellectual disability

participation and their carers. Those deemed incapacitated were included in the study in accordance to provisions of the English Mental Capacity Act 2005.

All recruitment and assessments at T2 were completed by one of three investigators (two medical doctors and one psychologist) between October 2007 and May 2008. The mean length of time between T1 and T2 was 2.9 years (34.3 months; range 25 to 45 months). The three interviewers were blind to the baseline diagnostic status.

\section{Screening stage}

Informants and all able participants completed a screen for decline in cognitive function and activities of daily living (ADL). Screen positives were defined as those who fulfilled any of the following conditions: a score at or above the single administration cognitive score thresholds for dementia for severe ( $\geq 34$ ), moderate ( $\geq 25$ ) and mild intellectual disability $(\geq 7)$ on the Dementia Questionnaire for Persons with Mental Retardation (DMR) (15); decline in ADL in more than three aspects which were not accounted for by physical health; or a delayed recall after ten minutes of fewer than two items in a 3-item memory task. Those screening negative were presumed not to have dementia.

\section{$\underline{\text { Informant interview }}$}

This comprised:

1. Details of the participant's current health and medications, any sensory impairment, information about level of functioning in early life.

2. A brief ADL schedule was based on a well-known scale (16) and in addition, informants were asked whether aspects of ADL had declined since the person was last seen for the initial study as well as possible explanations for the decline. The schedule included six self-care items (toileting, dressing, 
Dementia criteria in intellectual disability

bathing, mobility, and feeding), and nine instrumental ADL items (writing, reading, simple arithmetic ability, using money, room cleaning, safety awareness, food preparation, comprehension, and communication). Higher scores indicate better functioning.

3. The cognitive scale of the DMR (15). Higher scores indicate worse cognitive functioning.

4. Mental disorders and psychiatric symptoms were screened for using the PASADD Checklist (Revised) (17), a reliable tool for assessing adults with intellectual disability.

\section{Participant interview.}

Participants who had sufficient communication ability completed a three-item object memory task based on the Shoe Box Test (18).

\section{Assessment of people who screened positive}

In order to elicit symptoms of dementia, informants completed a questionnaire based on the Cambridge Mental Disorders Examination (CAMDEX) informant questionnaire (19). Participants who were sufficiently able completed the Test for Severe Impairment (TSI; (20)) encompassing several cognitive domains and used in adults with ID. Those who scored at the ceiling of the TSI were offered the Mini Mental State Examination (21), a widely used brief test for cognitive function. The Supermarket Fluency task (22) and the Tower of London test (23) were used to elicit verbal fluency and executive functioning. A structured physical examination was conducted by the researchers to record neurological symptoms and to identify other physical disoders relevant to the differential diagnosis of dementia.

\section{Blinding}


Dementia criteria in intellectual disability

In order to avoid observer bias, investigators and clinicians involved in the assessment and diagnostic phases of the study at T2 were blind to initial diagnosis or clinical and psychometric assessments at T1.

\section{Diagnosis}

At both T1 and T2, anonymized interview schedules for each participant were presented to two of three psychiatrists with expertise in either ID or old-age psychiatry (A.H., A.S. or G.L.) for independent diagnosis using an operationalized criteria tick list for each of the $I C D-10, D S M-I V$ and $D C-L D$ dementia criteria $(13,14)$. Participants were also rated against the criteria for dementia with Lewy bodies (DLB) (24) and frontotemporal dementia (FTD) (25). If they satisfied at least one of these criteria (i.e. ICD-10, DSM-IV, DC-LD main dementia criteria, or DLB or FTD criteria), they were considered to have dementia at T2.

Any disagreement in ratings was settled by discussion with the third psychiatrist. These disagreements were recorded for the purpose for evaluating inter-rater reliability. The following diagnostic principles were applied:

a) Dementia diagnosis required a decline in cognitive function from an individual's baseline rather than from general population norms (26).

b) A hierarchical process was followed, whereby developmental level, mental retardation syndrome, autistic disorders, physical illness and medication effects, sensory loss, environmental change or events, or mental illness were considered sequentially as possible reasons for screening positive. 
Dementia criteria in intellectual disability

c) The diagnosis of dementia was made in the presence of depressive symptoms or a history of stable mental illness if these were deemed not to account for the cognitive decline, depending on how the diagnostic criteria were worded. Dementia was not diagnosed in the presence of acute mental health problems.

\section{Diagnostic groups at T1}

During analysis the dementia status at T1 was unblinded and we defined ' $\mathrm{MCl}$ ' as those who screened positive at T1 but did not fulfil any of the three main dementia criteria. A "dementia" group had met at least one set of dementia criteria (ICD-10, DSM-IV, or DC-LD) at T1. The rest of the participants were a "no dementia" group. We also distinguished between DSM-IV, ICD-10 and DC-LD dementia.

\section{Diagnostic groups at T2:}

In terms of validity of the diagnoses made at $\mathrm{T} 1$, we combined those that had died or were diagnosed with any dementia at T2 in a 'poor outcome' group. Those who did not satisfy any of the dementia criteria (including those defined as $\mathrm{MCl}$ cases at T2) were considered a 'good outcome' group.

\section{Analysis}

Data analyses were performed using the Statistical Package for the Social Sciences (SPSS) Version 14.0 for Windows. Two-sided independent sample t-test was used to test for differences in continuous dependent variables between groups for parametric data. Chi square test and Kappa ( $\mathrm{K}$ ) statistics was used for categorical dependent variables. and inter-rater agreement respectively. Landis and Koch's classification 
Dementia criteria in intellectual disability

$(0.41-0.60=$ moderate $; 0.61-0.80=$ substantial $; \geq 0.81=$ almost perfect agreement; (27)) was used to classify agreement.

We examined deterioration in terms of change in ADL score after calculating a total score for each participant at T1 and T2 by summating the individual ADL domain scores from the brief ADL schedule. The mean change of score from T1 to T2 was compared between the groups. We examined in detail those who were no longer cases of dementia at T2 to understand diagnostic issues.

\section{Results}

\section{Participant demographics}

The recruitment process is summarised in diagram 1. $38(17.1 \%)$ of the original sample had died by T2. Those who had died were significantly older than the rest (75.5 v. 70.5 years, $t=-2.867, \mathrm{df}=43.402, p=0.006$, Mean difference $=5.03(95 \%$

$\mathrm{Cl}$ of difference $=1.50$ to 8.55$)$, SE of difference $=1.75)$. Of 184 remaining participants, 14 were uncontactable, and there were 16 refusals, leaving 154 (83.7\%) participants at T2. T2 participants did not differ from non-participants in sex $\left(X^{2}=\right.$ $1.140, \mathrm{df}=1, p=0.286)$ and level of intellectual disability $\left(X^{2}=1.204, \mathrm{df}=1, p=\right.$ 0.272). However, they were significantly older (70.9 v. 68.3. years, $t=1.983, \mathrm{df}=182$, $p=0.049$, Mean difference $=2.59(95 \% \mathrm{Cl}$ of difference $=0.13-5.16)$, SE of difference $=1.31$.

One participant was exluded due to a lack of collateral information leaving 191 participants for analyses that included those that had died, or 153 participants in analyses of living participants. 76 (49.4\%) participants were male; 86 (55.8\%) had mild ID and the rest had moderate to severe ID. At T1, median ADL scores were 43 (range $5-68 ; n=214$ ) while at $T 2$, the median was 39 (range $6-70 ; n=152$ ). At $T 1$, 
Dementia criteria in intellectual disability

the median DMR cognitive scale score was 10 (range $0-42$ ) and at T2, it was 8 (range 0-44). Further information on ADL and DMR scores at T1 and T2 are available in another paper (34). 65 (42.2\%) participants screened positive and $34(22.1 \%)$ met at least one of $I C D-10, D S M-I V$ and $D C-L D$ main dementia or the FTD or DLB criteria at T2.

\section{$\underline{\text { Inter-rater reliability }}$}

All three sets of main dementia criteria showed at least 'substantial' inter-rater agreement $(\mathrm{ICD}-10 \kappa=0.70, \mathrm{p}<0.01, \mathrm{~N}=64$; DSM-IV $\kappa=0.68, \mathrm{p}<0.01, \mathrm{~N}=64$; DC-LD $k=0.81, p<0.01, N=64)$, with the DC-LD in the category 'near perfect' inter-rater agreement.

\section{$\underline{\text { Stability of dementia diagnoses }}$}

Diagram 2 summarises changes in screening/diagnostic status from T1 to T2. Of the 27 people diagnosed with dementia at T1, 9 (33\%) had died. Of the 18 who remained, $12(67 \%)$ were diagnosed with dementia again at T2. Two of the six who no longer met dementia criteria became 'MCl' cases; the remaining four were not diagnosed with dementia (table 1). 75 (57\%) of those who were deemed not to have dementia at T1 remained dementia free at $\mathrm{T} 2 ; 10(33 \%)$ in the $\mathrm{T} 1$ ' $\mathrm{MCl}$ ' group became "no dementia" cases at T2. A similar proportion of those who did not have dementia at T1 converted to dementia.

\section{Predictive validity of dementia criteria in ID}

The odds ratio of having died by $\mathrm{T} 2$ for those diagnosed with dementia at T1 compared to the "no dementia" group was $2.85(95 \% \mathrm{Cl} 1.12-7.23$; Chi square = 5.156, df $=1 ; p=0.023)$; and for those with "MCl" it was $2.44(95 \% \mathrm{Cl} 0.98-6.09$; Chi square $=3.827, d f=1 ; p=0.050)$. 
In order to assess predictive validity of dementia criteria, we also compared "poor outcomes" at T2 (i.e. either dying or being re-diagnosed with dementia) between those diagnosed with dementia at T1 and those who did not have dementia (diagram 2). The 'dementia' group were more likely to have a 'poor outcome' compared to those without dementia (OR 9.18; 95\% Cl $3.43-24.53$; Chi square $=24.538 ; \mathrm{df}=1$, $\mathrm{p} \leq 0.001)$, while the 'MCl' group were not $(\mathrm{OR}=2.01 ; 95 \% \mathrm{Cl} 0.89-4.53$; Chi square $=2.859 ; \mathrm{df}=1, \mathrm{p}=0.091)$. The three sets of criteria had similar sensitivity and specificity (table 1). Specificity of having any dementia diagnosis at T1 was $95 \%$ and sensitivity was $30 \%$ (Table 1). When the participants who had died were excluded from the analysis, specificity of being re-diagnosed with dementia at T2 when they had any dementia diagnosis at $\mathrm{T} 1$ was $94.2 \%$, while sensitivity was $36.4 \%$.

Further evidence of predictive validity is provided by comparing change in Activity of Daily Living (ADL). The 'MCl' group showed a mean improvement in total combined ADL score between the initial study and follow up while the "no dementia" group showed a mean deterioration (1.00 vs -3.32 ; mean difference $=4.32$; SE of difference $=$ $1.68, \mathrm{df}=128 ; \mathrm{t}=-2.579 ; \mathrm{p}=0.011)$. Dementia cases at $\mathrm{T} 1$ had a significantly greater mean deterioration in ADL score than other participants (-7.67 vs. -2.66 ; mean difference $=5.00 ;$ SE of difference $=1.85, d f=147 ; t=2.706 ; p=0.008)$.

\section{$\underline{\text { Reasons for diagnostic instability }}$}

The six people who were diagnosed at T1 with dementia but were not re-diagnosed by any criteria at T2 are summarised in Table 2. Four had moderate to severe ID, or additional sensory disability (table 2). Four of these six participants were judged "false negatives" at T2 and two were "false positives" at T1. The false negative cases all had convincing dementia diagnoses at $\mathrm{T} 1$ and met the requirements for all three sets of criteria, but were either missed during screening or diagnosed as an $\mathrm{MCl}$ 
case at T2. Two of these participants showed further decline on tests or informant history between $\mathrm{T} 1$ and $\mathrm{T} 2$; one of whom was missed during screening as the informant did not report change. The other participant could not be diagnosed with dementia because although the informant reported decline in ADLs, memory problems were not noticed. The remaining two people did not demonstrate further decline, but were diagnosed with vascular dementia at $\mathrm{T} 1$, which often has periods of stability. We concluded that these four participants were not diagnosed with dementia at T2 due to inconsistent or unreliable information.

The two participants who were judged to be false positives at T1 were both diagnosed only with DSM-IV criteria. In these cases, evidence for memory deterioration was based on findings of the assessment at T1, but informants did not specifically report memory decline (Table 2).

\section{Discussion}

This is the first study to examine the validity and reliability of the diagnosis of dementia in ID adults and we found that the ICD-10, DSM-IV and DC-LD main dementia criteria all showed substantial inter-rater reliability, specificity and predictive validity in that they predicted 'poor outcomes' and deterioration. Sensitivity was expected to be poor as it is affected by incident cases at follow-up. The dementia diagnosis was, however, less stable than in the general population (28) (29) and this can be accounted for by variable quality of informant reports; difficulties in the assessment of those with moderate and severe intellectual disability or sensory impairments; or difficulties in detecting protracted periods of plateau in vascular dementia and the 'floor effect' in advanced dementia in the absence of longitudinal information. $\mathrm{MCl}$, as defined in this study, did not predict conversion to dementia, 
Dementia criteria in intellectual disability

and was therefore not comparable to the diagnosis in the general population, although clearly an indicator of ill health.

\section{$\underline{\text { Strengths and limitations }}$}

This study was a follow up to the largest survey of dementia in the intellectual disability population to date. We identified all older adults known to have intellectual disability in our catchment area and achieved high participation rates. We included a representative sample of adults with varying levels of ability, including those who lacked capacity to consent to taking part, as they were likely to be those with most disability and needs. Our drop-out rate was low, and we were able to interview $84 \%$ of the survivors from baseline.

Those who screened positive were fully assessed before we applied a rigorous and standardised diagnostic procedure. Blinding to the original assessment and diagnostic status at T2 ensured that we eliminated observer bias. However, this meant that the follow up was in fact a cross-sectional assessment, which is known to be less reliable than sequential assessments. Lastly, there was no post mortem validation of the diagnosis of dementia in any of the deceased participants. We may therefore have under- or overestimated validity.

\section{$\underline{\text { Inter-rater reliability }}$}

All three sets of main dementia criteria showed at least substantial inter-rater reliability. Our reliability figures for the ICD-10 and DSM-IV sets of criteria were comparable to those found in the general older adult population (30) (31).

\section{Validity and reliability of dementia diagnoses}

Dementia is usually a progressive condition. If a set of diagnostic criteria for dementia were valid, one would expect those it identifies as having dementia over a 
Dementia criteria in intellectual disability

period of time to have on average poorer outcomes, including higher death rates and greater deterioration in ADL, than those without dementia. This was indeed our finding for each of the standardised criteria.

Those identified as having dementia should still have dementia when assessed again later. We found in our study a small proportion (7\%) went from 'dementia' to 'MCl' and a significant minority (15\%) did not reach the screening thresholds for full assessment at follow up, which were more than equivalent diagnostic errors in the general population (32). Closer examination of these cases revealed that the sensitivity of the screening process was affected by quality of information provided by informants. In clinical practice information from previous assessments (to which we were blinded) would have been available at the second assessment. This would have avoided having negative screens because of lack of information.

General population studies have shown that the DSM-IV criteria are more inclusive in the diagnosis of dementia than the ICD-10 (3), despite relatively minor differences (33). We have previously shown that the DSM-IV dementia criteria were also more inclusive than the ICD-10 in older adults with ID (14). The ICD-10 criteria excluded those with mild dementia and a considerable proportion of those with moderate to severe dementia because of specifying the presence of emotional and behavioural symptoms. The current study showed that the DSM-IV criteria may occasionally identify 'false positive' cases of dementia in individuals with pre-existing cognitive deficits.

\section{Implications for forthcoming ICD and DSM revisions}

Populations with pre-existing cognitive deficits such as those with ID are a litmus test for the validity of dementia criteria, and our findings have implications for the ICD-11 and DSM-V revisions currently under way. 
ICD revisions should reconsider the requirement for behavioural and emotional changes as these symptoms were poor at discriminating between those with and without dementia in adults from the general population and those with ID $(2,14)$. A substitiute could be requirement for change in functioning (similar to the DSM-IV and DSM-V), as this was better than cognitive symptoms at identifying those with dementia in adults with more severe levels of intellectual disability (34).

The forthcoming DSM revision could be more specific about the sources of information required to demonstrate a decline in memory. Informant report of cognitive decline should be sought and taken into account when standard cognitive testing is difficult, and dementia criteria may need to specify that change in function and cognition should be compared to an individual's own baseline. These issues have been addressed in the proposed criteria for "Major Neurocognitive Disorder" of the DSM-V, which may therefore have improved validity in populations with preexisting cognitive deficits compared with the DSM-IV.

In this study, $\mathrm{MCl}$ was defined broadly as those performing worse than expected on a memory test, or having significant functional decline or a positive DMR screen. The conversion rate from "MCl" to dementia of $13 \%$ over 2.9 years was not markedly different from that of a 'normal' state, and was considerably lower than the $60 \%$ per 5 year conversion rate of $\mathrm{MCl}$ cited in the general population (4). However, many of the $\mathrm{MCl}$ cases in our study who died may have had dementia, and the rate of 'MCl' cases reverting to 'normal' at follow up was similar to $\mathrm{MCl}$ cited in the general population (36). Furthermore, the conversion rate of "no dementia" cases was very high compared to the general population. Nevertheless, our definition of $\mathrm{MCl}$ in the ID population have identified a heterogeneous group with co-morbid conditions. This problem has also been noted in the general population, where various definitions of 
Dementia criteria in intellectual disability

$\mathrm{MCl}$ result in very different prevalence rates $(35,37)$. $\mathrm{MCl}$ definitions may need to be more specific in the ID population, e.g. cognitive decline on formal testing, or on reliable informal report, relative to the individual's premorbid functioning, not sufficient to impair everyday function and not sufficient to fulfil any of the main dementia criteria.

Word count 3611 


\section{Reference List:}

1. Stevens T, Livingston G, Kitchen G, et al: Islington study of dementia subtypes in the community. Br J Psychiatry 2002; 180:270-276

2. Wancata J, Borjesson-Hanson A, Ostling S, et al: Diagnostic criteria influence dementia prevalence. Am J Geriatr Psychiatry 2007; 15:1034-1045 3. Erkinjuntti T, Ostbye T, Steenhuis R, et al: The effect of different diagnostic criteria on the prevalence of dementia. The New England journal of medicine 1997; 337:1667-1674

4. Morris JC, Storandt M, Miller JP, et al: Mild cognitive impairment represents early-stage Alzheimer disease. Archives of neurology 2001; 58:397-405

5. Palmer K, Backman L, Winblad B, et al: Mild cognitive impairment in the general population: occurrence and progression to Alzheimer disease. Am J Geriatr Psychiatry 2008; 16:603-611

6. WHO: The ICD-10 Classification of Mental and Behavioural Disorders Geneva World Health Organization, 1992

7. American Psychiatric A, American Psychiatric A,Task Force on D-I: Diagnostic and statistical manual of mental disorders DSM-IV-TR, Washington, DC, American Psychiatric Association, 2000

8. Royal College of P: DC-LD : diagnostic criteria for psychiatric disorders for use with adults with learning disabilities/mental retardation, Royal College of Psychiatrists, 2001

9. Beeri MS,Goldbourt U: Late-Life Dementia Predicts Mortality Beyond Established Midlife Risk Factors. American Journal of Geriatric Psychiatry Publish Ahead of Print:10.1097/JGP.1090b1013e3181e1043d1090

10. Rait G, Walters K, Bottomley C, et al: Survival of people with clinical diagnosis of dementia in primary care: cohort study. BMJ (Clinical research ed 2010; $341: c 3584$

11. Gurland B, Golden RR, Teresi JA, et al: The SHORT-CARE: an efficient instrument for the assessment of depression, dementia and disability. Journal of gerontology 1984; 39:166-169

12. O'Connor DW, Pollitt PA, Jones BJ, et al: Continued clinical validation of dementia diagnosed in the community using the Cambridge Mental Disorders of the Elderly Examination. Acta psychiatrica Scandinavica 1991; 83:41-45

13. Strydom A, Hassiotis A, King M, et al: The relationship of dementia prevalence in older adults with intellectual disability (ID) to age and severity of ID. Psychological medicine 2009; 39:13-21

14. Strydom A, Livingston $G$, King M, et al: Prevalence of dementia in intellectual disability using different diagnostic criteria. Br J Psychiatry 2007; 191:150-157

15. Evenhuis HM: Further evaluation of the Dementia Questionnaire for Persons with Mental Retardation (DMR). Journal of Intellectual Disability Research 1996; 40:369-373

16. Lawton MP,Brody EM: Assessment of older people: self-maintaining and instrumental activities of daily living. The Gerontologist 1969; 9:179-186 
17. Moss $\mathrm{S}$, Prosser $\mathrm{H}$, Costello $\mathrm{H}$, et al: Reliability and validity of the PASADD checklist for detecting psychiatric disorders in adults with intellectual disability. Journal of Intellectual Disability Research 1998; 42:173-183 18. Burt DB,Aylward EH: Test battery for the diagnosis of dementia in individuals with intellectual disability. Journal of Intellectual Disability Research 2000; 44:175-180

19. Ball SL, Holland AJ, Huppert FA, et al: The modified CAMDEX informant interview is a valid and reliable tool for use in the diagnosis of dementia in adults with Down's syndrome. Journal of Intellectual Disability Research 2004; 48:611-620

20. Albert M,Cohen C: The Test for Severe Impairment - An Instrument for the Assessment of Patients with Severe Cognitive Dysfunction. Journal of the American Geriatrics Society 1992; 40:449-453

21. Folstein MF, Folstein SE,McHugh PR: "Mini-mental state". A practical method for grading the cognitive state of patients for the clinician. J Psychiatr Res 1975; 12:189-198

22. Troyer AK: Normative data for clustering and switching on verbal fluency tasks. J.Clin.Exp.Neuropsychol. 2000; 22:370-378

23. Shallice T: Specific Impairments of Planning. Philosophical Transactions of the Royal Society of London Series B-Biological Sciences 1982; 298:199-209

24. McKeith IG, Galasko D, Kosaka K, et al: Consensus guidelines for the clinical and pathologic diagnosis of dementia with Lewy bodies (DLB): Report of the consortium on DLB international workshop. Neurology 1996; 47:11131124

25. McKhann GM, Albert MS, Grossman M, et al: Clinical and pathological diagnosis of Frontotemporal Dementia - Report of the work group on Frontotemporal Dementia and Pick's disease. Archives of neurology 2001; 58:1803-1809

26. Aylward EH, Burt DB, Thorpe LU, et al: Diagnosis of dementia in individuals with intellectual disability. Journal of Intellectual Disability Research 1997; 41:152-164

27. Landis JR,Koch GG: The measurement of observer agreement for categorical data. Biometrics 1977; 33:159-174

28. Brunnstrom $\mathrm{H}$,Englund $\mathrm{E}$ : Clinicopathological concordance in dementia diagnostics. Am J Geriatr Psychiatry 2009; 17:664-670

29. Ranginwala NA, Hynan LS, Weiner MF, et al: Clinical criteria for the diagnosis of Alzheimer disease: still good after all these years. Am J Geriatr Psychiatry 2008; 16:384-388

30. Knopman DS, DeKosky ST, Cummings JL, et al: Practice parameter: Diagnosis of dementia (an evidence-based review) - Report of the Quality Standards Subcommittee of the American Academy of Neurology. Neurology 2001; 56:1143-1153

31. Baldereschi M, Amato MP, Nencini P, et al: Cross-National Interrater Agreement on the Clinical Diagnostic-Criteria for Dementia. Neurology 1994; 44:239-242

32. Bermejo-Pareja F, Benito-Leon J, Vega S, et al: Consistency of clinical diagnosis of dementia in NEDICES: A population-based longitudinal study in Spain. Journal of geriatric psychiatry and neurology 2009; 22:246-255 
33. First MB: Harmonisation of ICD-11 and DSM-V: opportunities and challenges. Br J Psychiatry 2009; 195:382-390

34. Jamieson-Craig R, Scior K, Chan T, et al: Reliance on Carer Reports of Early Symptoms of Dementia Among Adults With Intellectual Disabilities. Journal of Policy and Practice in Intellectual Disabilities 2010; 7:34-41 35. Ganguli M, Chang CC, Snitz BE, et al: Prevalence of Mild Cognitive Impairment by Multiple Classifications: The Monongahela-Youghiogheny Healthy Aging Team (MYHAT) Project. Am J Geriatr Psychiatry 2010; 18:674683

36. Larrieu S, Letenneur L, Orgogozo JM, et al: Incidence and outcome of mild cognitive impairment in a population-based prospective cohort. Neurology 2002; 59:1594-1599

37. Kochan NA, Slavin MJ, Brodaty $\mathrm{H}$, et al: Effect of Different Impairment Criteria on Prevalence of "Objective" Mild Cognitive Impairment in a Community Sample. Am J Geriatr Psychiatry 2010 18:711-722 